\title{
Sleeping During the First Wave: Demographic, Psychological, Chronobiological, and Work-Related Predictors of Sleep Disturbances Throughout the COVID-19 Lockdown
}

\author{
Federico Salfi \\ University of L'Aquila \\ Marco Lauriola \\ Sapienza University of Rome \\ Aurora D'Atri \\ University of L'Aquila \\ Giulia Amicucci \\ Sapienza University of Rome \\ Lorenzo Viselli \\ University of L'Aquila \\ Daniela Tempesta \\ University of L'Aquila \\ Michele Ferrara ( $\nabla$ michele.ferrara@univaq.it) \\ University of L'Aquila
}

\section{Research Article}

Keywords: COVID-19, population, lockdown

Posted Date: January 22nd, 2021

DOI: https://doi.org/10.21203/rs.3.rs-145211/v1

License: (c) (i) This work is licensed under a Creative Commons Attribution 4.0 International License.

Read Full License 


\section{Abstract}

The first COVID-19 contagion wave caused unprecedented restraining measures worldwide. In Italy, a period of generalized lockdown involving home confinement of the entire population was imposed for almost two months (9 March-3 May 2020). The present is the most extensive investigation aimed to unravel the demographic, psychological, chronobiological, and work-related predictors of sleep disturbances throughout the pandemic emergency.

A total of 13989 Italians completed a web-based survey during the confinement period ( 25 March-3 May). We collected demographic and lockdown-related work changes information, and we evaluated sleep quality, insomnia and depression symptoms, chronotype, perceived stress, and anxiety using validated questionnaires.

The majority of the respondents reported a negative impact of confinement on their sleep and a delayed sleep phase. We highlighted an alarming prevalence of sleep disturbances during the lockdown. Main predictors of sleep disturbances identified by regression models were: female gender, advanced age, being a healthcare worker, living in southern Italy, confinement duration, and a higher level of depression, stress, and anxiety. The circadian evening preference turned out as a vulnerability factor, while morningtype individuals showed a lower predisposition to sleep and psychological problems. Finally, working from home emerged as a protective factor against the development of sleep disturbances.

Besides confirming the role of specific demographic and psychological factors in developing sleep disorders during the COVID-19 pandemic, we demonstrated circadian typologies react differently to a particular period of reduced social jetlag. Finally, our results indicated working from home promotes wellbeing during the current pandemic period.

\section{Introduction}

The rapid spread of the new Coronavirus (SARS-CoV-2) outbreak led the global governments to adopt generalized lockdown and social distancing measures to limit the virus propagation. The Italian population was subjected to home confinement for almost two months (9 March-3 May 2020). The restraining measures involved unprecedented limitations of mobility rights and social interactions. This stressful situation profoundly compromised the general population's everyday life, resulting in pervasive psychological repercussions[1, 2]. In this extraordinary historical period, sleep could represent one of the primary victims[3]. Several aspects could contribute to sleep disturbances during this period. The fear of contagion, the uncertainty of the future, the low activity levels during the day, and the reduction of social interactions could all be factors associated with developing sleep problems. Notably, the home confinement disrupted the daily activity routine, compromising the daylight exposure, which is a crucial regulator of the circadian rhythms. Several cross-sectional and longitudinal studies confirmed this assumption, showing a high prevalence of sleep disturbances during the lockdown period (for a meta- 
analysis[4]). Meanwhile, there was a worldwide increase in electronic backlit screen exposure, which was suggested as a mediator of sleep deterioration in the long run[5].

In the current study, we present the most extensive investigation $(\mathrm{N}=13989)$ aimed to provide a comprehensive snapshot of sleep health and habits during the entire home confinement period due to the COVID-19 outbreak. We used a web-based survey covering the two months of total lockdown to evaluate sleep quality (by means of the Pittsburgh Sleep Quality Index - PSQI[6, 7]), insomnia symptoms (Insomnia Severity Index - ISI[8, 9]), chronotype (reduced version of the Morningness-Eveningness Questionnaire MEQr[10]), depression symptoms (Beck Depression Inventory-second edition - BDI-II[11]), perceived stress (10-item Perceived Stress Scale - PSS-10[12]), and anxiety (state-anxiety subscale of the State-Trait Anxiety Inventory - STAI-X1[13]) of the Italian population under restraining measures. The present investigation was conceived to understand the sociodemographic, psychological, chronobiological, and work-related predictors of the sleep disturbances during the home confinement period.

Several cross-sectional studies showed women[14-18] and healthcare workers[19, 20] as the categories suffering the most. Furthermore, literature also revealed a close relationship between psychological wellbeing and sleep disturbances during the pandemic period[4, 16, 21, 22]. We first of all expected to confirm these results within our larger sample.

Moreover, the present study aimed to provide new insights about some aspects not yet addressed, such as the role of the individual circadian preference (chronotype) and the lockdown-related work changes in the expression of sleep disturbances during the first COVID-19 outbreak wave.

The restraining measures impacted the sleep/wake rhythms. People delayed the bedtime and wake-up time during the lockdown[18,21,23,24], resulting in a reduction of social jetlag[23-25], which could be conceived as a proxy for a challenged circadian system and compromised sleep[26, 27]. Conventionally, three main chronotypes have been identified[28]: the morning-type (MT), the neither-type (NT), and the evening-type (ET). The ET typically showed lower sleep quality[29-31], and this evidence was ascribed to the misalignment between the biological and social clock (i.e., the timing of social obligations), which is associated to the unhealthy manifestation of social jetlag[26, 28]. Furthermore, higher levels of negative mood and anxiety characterize ET individuals[31-34]. The social jetlag and the sleep difficulties have been proposed to account for the ET's tendency to experience psychological symptoms[35-38]. In this view, we could expect that during a period of large-scale reduction of the social jetlag such as the home confinement due to the COVID-19 outbreak, the gap between circadian typologies could be narrowed down because ET, in particular, may have benefitted from a period marked by a loosening of rigorous sleep/wake schedule due to weaker social and working obligations.

Finally, the lockdown impacted the working routine of the majority of the population, leading to the suspension of the working activity and the imposition of home working. The widespread possibility of working from home in telematic mode with a great flexibility in the working schedule could be a further factor contributing to the general population's sleep quality. Therefore, we aimed at verifying the influence of changes in working activity caused by the restraining measures on sleep habits. 


\section{Results}

\section{Lockdown-related consequences on sleep and prevalence of sleep disturbances}

The majority of respondents reported a negative impact of the restraining measures on their sleep, delayed bedtime and wake-up time, and maintained unchanged nap habits. According to the PSQI and ISI criteria, over six out of ten of the participants were poor sleepers, and $15 \%$ of the respondents had symptoms of moderate/severe clinical insomnia (Table 1). 
Table 1

Prevalence of lockdown-related consequences and sleep disturbance prevalence within the total sample.

\begin{tabular}{|lc|}
\hline \multicolumn{2}{|c|}{ N (\%) } \\
\hline Perceived impact \\
\hline Negative & $8455(60.4)$ \\
\hline None & $3306(23.6)$ \\
\hline Positive & $2228(15.9)$ \\
\hline Bedtime & \\
\hline Anticipated & $1288(9.2)$ \\
\hline Unchanged & $4483(32.0)$ \\
\hline Delayed & $8218(58.7)$ \\
\hline Wake-up time & \\
\hline Anticipated & $1570(11.2)$ \\
\hline Unchanged & $3563(25.4)$ \\
\hline Delayed & $8856(63.3)$ \\
\hline Nap habits & \\
\hline Increased & $2477(17.7)$ \\
\hline Unchanged & $9045(64.6)$ \\
\hline Reduced & $2467(17.6)$ \\
\hline Sleep quality (PSQI) \\
\hline Poor & $8053(61.1)$ \\
\hline Good & $5122(38.8)$ \\
\hline Insomnia (ISI) & \\
\hline No & $6597(47.1)$ \\
\hline Subthreshold & $5297(37.8)$ \\
\hline Moderate & $1840(13.1)$ \\
\hline Severe & $255(1.8)$ \\
\hline
\end{tabular}


Significant regression equations were found with PSQI and ISI scores as dependent variables (PSQI: $\mathrm{R}^{2}=$ $0.30, F_{17,8552}=219.07, \mathrm{P}<0.001 ;$ ISI: $\mathrm{R}^{2}=0.35, \mathrm{~F}_{17,9046}=285.07, \mathrm{P}<0.001$ ).

As shown in Table 2, older age, female gender, healthcare work, living in southern Italy, and confinement duration were associated with highest PSQI and ISI scores. Moreover, lower education predicted poorer sleep quality. Lower scores of MEQr (pointing to evening chronotype), and a higher level of depression, perceived stress, and anxiety predicted poorer sleep quality and more severe insomnia symptoms. 
Table 2

Results ( $\beta$ and $P$ ) of the multiple linear regressions on PSQI and ISI scores.

\begin{tabular}{|c|c|c|c|c|}
\hline \multirow[b]{2}{*}{ Predictor } & \multicolumn{2}{|c|}{ PSQI score } & \multicolumn{2}{|c|}{ ISI score } \\
\hline & $\beta$ & $\mathrm{P}$ & $\beta$ & $\mathrm{P}$ \\
\hline Intercept & & $<0.001$ & & 0.129 \\
\hline Age & 0.136 & $<0.001$ & 0.048 & $<0.001$ \\
\hline \multicolumn{5}{|l|}{ Gender: } \\
\hline Woman & \multicolumn{2}{|c|}{ Reference } & \multicolumn{2}{|c|}{ Reference } \\
\hline Man & -0.074 & $<0.001$ & -0.035 & $<0.001$ \\
\hline \multicolumn{5}{|l|}{ Education: } \\
\hline Middle school & \multicolumn{2}{|c|}{ Reference } & \multicolumn{2}{|c|}{ Reference } \\
\hline High school & -0.115 & 0.003 & 0.002 & 0.957 \\
\hline Graduated & -0.160 & $<0.001$ & -0.046 & 0.224 \\
\hline Over graduated & -0.222 & $<0.001$ & -0.071 & 0.151 \\
\hline \multicolumn{5}{|l|}{ Occupation: } \\
\hline Health work & \multicolumn{2}{|c|}{ Reference } & \multicolumn{2}{|c|}{ Reference } \\
\hline Other work & -1.204 & $<0.001$ & -0.702 & 0.038 \\
\hline Student & -0.053 & $<0.001$ & -0.038 & $<0.001$ \\
\hline Unemployed & -0.307 & 0.088 & -0.142 & 0.404 \\
\hline \multicolumn{5}{|l|}{ Geographic location: } \\
\hline Northern Italy & \multicolumn{2}{|c|}{ Reference } & \multicolumn{2}{|c|}{ Reference } \\
\hline Central Italy & 0.026 & 0.903 & -0.142 & 0.487 \\
\hline Southern Italy & 0.699 & $<0.001$ & 0.294 & 0.054 \\
\hline Home confinement duration & 0.075 & $<0.001$ & 0.070 & $<0.001$ \\
\hline \multicolumn{5}{|l|}{ Forced quarantine: } \\
\hline Yes & \multicolumn{2}{|c|}{ Reference } & \multicolumn{2}{|c|}{ Reference } \\
\hline No & -0.636 & 0.142 & -0.072 & 0.859 \\
\hline No response & 0.051 & 0.386 & 0.093 & 0.097 \\
\hline MEQr score & -0.015 & $<0.001$ & -0.013 & $<0.001$ \\
\hline
\end{tabular}




\begin{tabular}{|lllll|}
\hline & \multicolumn{2}{ll}{ PSQI score } & \multicolumn{2}{l|}{ ISI score } \\
\hline BDI-II score & 0.027 & $<0.001$ & 0.030 & $<0.001$ \\
\hline PSS-10 score & 0.008 & $<0.001$ & 0.011 & $<0.001$ \\
\hline STAI-X1 score & 0.143 & $<0.001$ & 0.152 & $<0.001$ \\
\hline
\end{tabular}

Abbreviations. MEQr, Morningness-Eveningness Questionnaire-reduced; BDI-II, Beck Depression Inventorysecond edition; PSS-10, Perceived Stress Scale-10 item; STAI-X1, state-anxiety subscale of the State-Trait Anxiety Inventory.

a Northern Italy: Aosta Valley, Emilia Romagna, Friuli-Venezia Giulia, Liguria, Lombardy, Piedmont, Trentino-Alto Adige, and Veneto. ${ }^{b}$ Central Italy: Lazio, Marche, Tuscany, and Umbria. ${ }^{c}$ Southern Italy: Abruzzo, Apulia, Basilicata, Calabria, Campania, Molise, Sardinia, and Sicily.

\section{Chronotype differences}

According to the MEQr criteria, our sample consisted of 3261 MT subjects (21.3\%), 9181 NT (65.6\%), and 1547 ET (11.1\%). Chi-square tests (Table 3) showed significant associations of the chronotype group (MT, NT, ET) with the perceived impact of the restraining measures, with the reported changes in bedtime, wake-up time, and nap frequency, and with the prevalence of poor sleepers and clinical insomnia conditions. In particular, a higher proportion of ET subjects reported a negative impact of the restraining measures, delayed bedtime and wake up time, and changes of nap habits. Additionally, the ET group was marked by a higher prevalence of poor sleepers and clinical insomniacs. MT participants showed the opposite pattern of results. 
Table 3

Frequency of the lockdown-related perceived impact on sleep, reported changes of bedtime, wake-up time and nap habits, and prevalence of poor sleepers and clinical insomnia conditions within the three chronotype groups (MT, NT, ET). Chi-square test results are also reported $\left(\chi^{2}\right.$ and $\left.P\right)$.

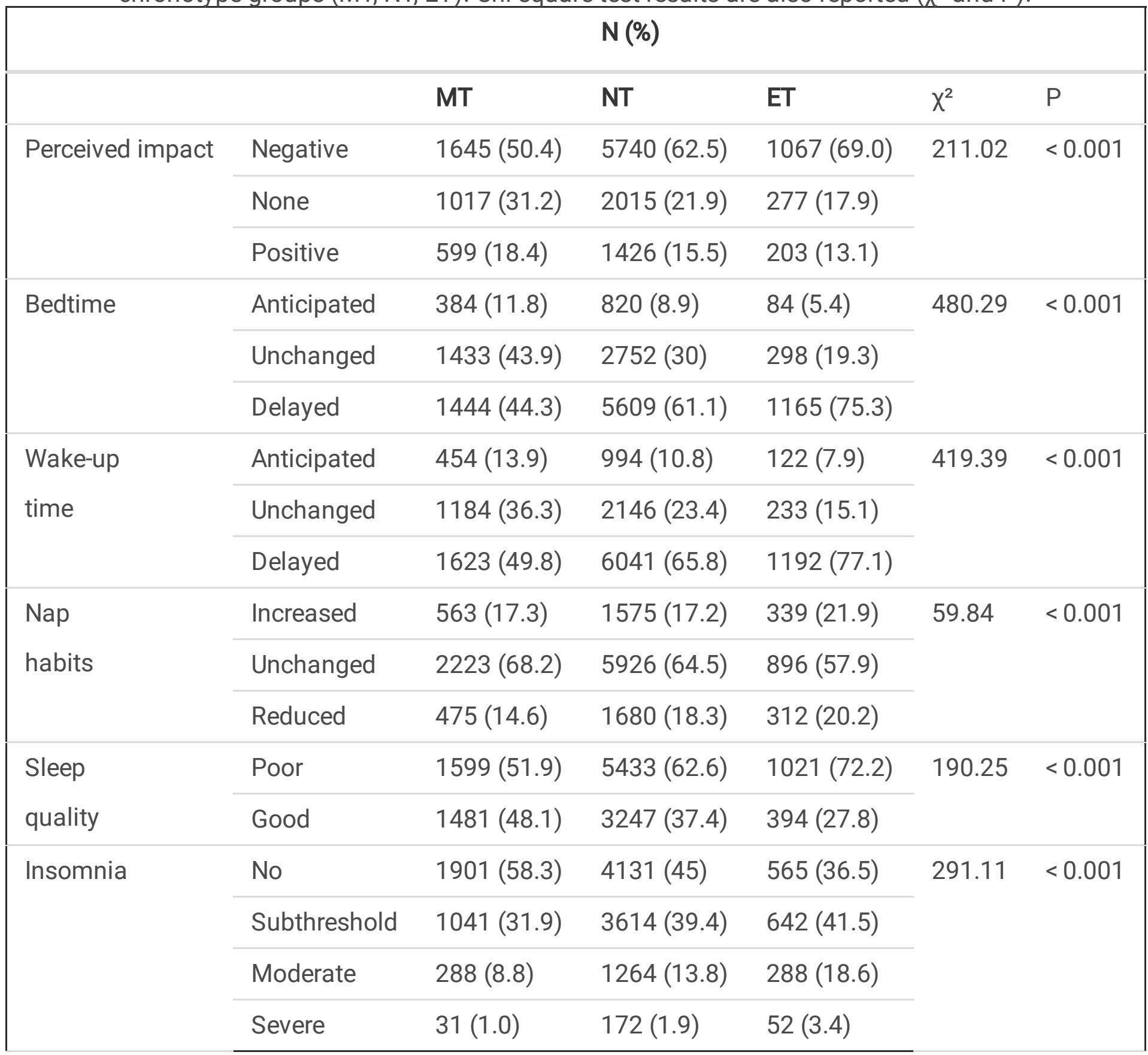

Abbreviations. MT, Morning-type; NT, Neither-type; ET, Evening-type.

As hypothesized, the MEQr scores and the age variable were highly correlated $(R=0.28 ; P<0.001)$, confirming the assumption of using the age as covariate in the subsequent analyses. One-way ANCOVAs carried out on sleep and psychological questionnaires showed a significant effect of the "chronotype" factor (MT, NT, ET) (PSQI: $F_{13171}=152.70, P<0.001$; ISI: $F_{13985}=173.01, P<0.001 ; B D I-I I: F_{9978}=134.17$, $P<0.001$; PSS-10: $F_{9278}=95.01, P<0.001 ;$ STAI-X1: $\left.F_{9060}=45.58, P<0.001\right)$. The "age" covariate yielded a significant effect in the analyses of PSQI, BDI-II, and PSS-10 scores (all P<0.001), while it was not significant for ISI $(P=0.35)$ and STAI-X1 ( $P=0.58)$. Post hoc comparisons (Fig. 1) showed that the ET 
group had higher scores in all the dimensions compared to MT and NT (all $\mathrm{P}<0.001$ ). NT showed higher scores compared to MT group for all the variables (all $\mathrm{P}<0.001$ ).

Exploratory analyses highlighted a significant difference between the three chronotype groups for the reported sleep duration $\left(F_{2,13986}=14.14, P<0.001\right)$. ET participants slept more (mean $\pm S E M, 444 \min \pm$ $2 \mathrm{~min}$ ) than NT (426 $\mathrm{min} \pm 1 \mathrm{~min})$ and MT groups (420 $\mathrm{min} \pm 1 \mathrm{~min}$; both $\mathrm{P}<0.001)$.

\section{Working activity changes}

A total of 3314 workers (38.9\% of the total workers' sample) suspended their working activity during the lockdown. T-tests on PSQI and ISI scores showed significant differences between the group of respondents that suspended and the group that preserved their working activity (PSQI: $t_{8084}=2.56, P=$ 0.01 ; ISI: $\left.\mathrm{t}_{8523}=6.18, \mathrm{P}<0.001\right)$. The suspension of the working activity was associated with lower sleep quality (mean $\pm S E M, 7.15 \pm 0.07$ vs. $6.91 \pm 0.05)$ and more severe insomnia symptoms $(8.67 \pm 0.10$ vs. $7.91 \pm 0.08)$.

Among the sample who continued to work (5211 subjects), a total of 3536 respondents worked from home, 2125 reported reduced working time, 1989 maintained unchanged the work duration, and 1097 subjects increased their daily working time.

Two-way ANOVAs on PSQI and ISI scores highlighted significant effects of "home working" (PSQI: $F_{1,4941}$ $=45.91, P<0.001$; ISI: $\left.F_{1,5205}=17.60, P<0.001\right)$, and "daily working time" factors (PSQI: $F_{2,4941}=28.49$, $P<0.001$; ISI: $F_{2,5205}=25.21, P<0.001$ ). The interactions between the two factors ("home working" $x$ "daily working time") were significant in both analyses (PSQI: $F_{2,4941}=6.23, P=0.002 ;$ ISI: $F_{2,5205}=4.13$, $P=0.02)$.

Post hoc comparisons (Fig. 2) pointed to lower sleep quality and more severe insomnia symptoms for the participants who increased the daily working time within both home working and regular working group (all $\mathrm{P}<0.01$ ). There were no differences in PSQI and ISI scores between the two groups (home vs. regular work) when they reduced the working time (both $P=1.00$ ). When the daily working time was the same or increased compared with the pre-outbreak period, the home workers showed lower scores on PSQI (both $P<0.001)$ and ISI questionnaires $(P=0.03, P=0.01$; respectively $)$. Notably, the home workers who increased the daily working time reported the same sleep quality and insomnia symptoms of the regular workers who reduced (PSQI: $\mathrm{P}=1.00 ; \mathrm{ISI}: \mathrm{P}=.25$ ) or maintained unchanged the working duration (PSQI: $\mathrm{P}$ $=1.00 ;$ ISI: $\mathrm{P}=.85$ ).

Exploratory analyses showed that the home working group went to bed and woke-up later (bedtime: hh:mm \pm SEM, 00:01 $\pm 1 \mathrm{~min}$; wake-up time: 08:17 $\pm 1 \mathrm{~min}$ ) and slept more (419 $\mathrm{min} \pm 1 \mathrm{~min}$ ) compared to the respondents who continued to reach the workplace (bedtime: $23: 33 \pm 2 \mathrm{~min}, \mathrm{t}_{5209}=11.93, \mathrm{P}<0.001$; wake-up time: 07:28 $\pm 2 \mathrm{~min}, \mathrm{t}_{5209}=19.20, \mathrm{P}<0.001$; sleep duration: $392 \mathrm{~min} \pm 2 \mathrm{~min}, \mathrm{t}_{5209}=11.38, \mathrm{P}<$ $0.001)$. 


\section{Discussion}

Our study provided a comprehensive description of sleep health during the lockdown in Italy. The majority of respondents (approximately 60\%) declared a negative impact of the restraining measure and delayed bedtime and wake-up time. We highlighted an alarming prevalence of poor sleepers and clinical insomniacs within our large sample: most of the participants were poor sleepers and presented insomnia symptoms from subthreshold to severe. In line with the current literature[4, 16, 21, 22], we demonstrated a strong relationship between sleep variables and depression, stress, and anxiety. We confirmed the results of other cross-sectional investigations on healthcare workers[19, 20] and women[14-18], which appeared as the categories experiencing the most severe sleep problems during lockdown worldwide. Furthermore, advanced age predicted more severe sleep disturbances. An interpretation of the healthcare workers' results is related to the well-known increased stressful workload, accompanied by higher contagion risk. Consistently, several studies demonstrated a high prevalence of post-traumatic stress disorder (PTSD) symptoms and mental health problems among the healthcare operators during the COVID-19 pandemic[20,39]. On the other hand, we suggest caution in the interpretation of the results on women and elderly population since these two factors were typically associated with the poorest sleep quality[40, 41] and the higher predisposition to insomnia conditions even in the pre-outbreak period[42, 43]. Consistently, our recent longitudinal study[44] showed that the time course of sleep disturbance was different between men and women, and the male gender appeared as the most vulnerable to the prolongation of the restraining measures.

We highlighted more severe sleep disturbances in southern Italy, and this result is inconsistent with the available literature on sleep and COVID-19 in the Italian population[17], which identified a higher prevalence of sleep problems in northern Italy. However, differences in the period under consideration might explain the different results. The previous investigation[17] was referred to the first weeks of the outbreak, when the geographic propagation of the contagion in Italy was extremely unbalanced towards northern regions[45]. Our study covered the entire confinement period instead, providing a more reliable overview of the effect of the pandemic propagation in southern Italy. Moreover, we hypothesize that our results could also reflect the pandemic's economic consequences since southern Italy was the territorial area most affected economically by the COVID-19 crisis[46]. Finally, the confinement duration was a predictive factor of sleep disturbances, corroborating the hypothesis of a cumulative detrimental effect of the protracted lockdown period. However, scarce evidence had been provided worldwide, with only a few studies addressing this question through longitudinal investigations across the confinement period[5, 44, 47].

As far as the influence of chronotypes is concerned, according to the initial hypothesis, ET respondents reported the most prevalent delay of the sleep phase. These results are consistent with another Italian cross-sectional study carried out during the lockdown[48]. However, the ET participants reported suffering more from the confinement situation than the other circadian typologies. Coherently, this group showed the lowest sleep quality and the highest level of insomnia, depression, perceived stress, and anxiety. Meanwhile, MT showed the opposite pattern of results, declaring a lower negative impact of the 
restraining measures and a higher prevalence of preserved sleep schedule. This finding was exemplified by the highest sleep quality, less marked insomnia and depression symptoms, and the lowest perceived stress and anxiety levels.

The present results pointed to a particular vulnerability of the ET group, although the lockdown was a favourable period to reduce the mismatch between internal and social clocks. Of note, the present results are consistent with those obtained during the pandemic period in an adolescent clinical population[49].

Our findings suggest that the well-known higher predisposition to sleep disturbances of the ET people[29-31] should not be considered only as a consequence of the accumulated sleep debt due to social and working obligations. In fact, during an unprecedented period that unlocked time for sleep[27], the ET respondents paradoxically reported the longest sleep time, however preserving the more severe sleep problems. This evidence suggests that the evening-individuals' sleep disturbances may instead originate from the misalignment of the delayed sleep pattern to the biological night[50].

On the other hand, the morning circadian preference emerged as a protective factor, both on the sleep and psychological sides. Recent studies demonstrated that the three chronotype groups differ for resilience level[51-53] and perceived stress[54]. The morning chronotype seems to be more able to cope with challenging situations, while ET individuals are more predisposed to develop PTSD [55, 56]. Our results confirm this assumption, even in a context of reduced social jetlag, such as during the lockdown.

As expected, a substantial percentage of participants suspended the working activity during the lockdown (38.9\%), leading to a lower sleep quality and more severe insomnia symptoms. We believe that these findings can be ascribable to the economic repercussions of the work interruption, although this dimension was not assessed in the present study. However, the possibility of maintaining a regular working activity during the confinement could have had a direct positive impact on preserving sleep health. The absence of a daily activity routine could emphasize the sense of boredom, leading to a slowing of the felt pace of the time flow[57]. Consistently, a recent study demonstrated a relationship between the increase of sleep difficulties and the feeling of time dilatation during the lockdown period[21]. Coherently, in our study, the unemployed participants were the only group that did not differ from the healthcare workers for sleep disturbances.

On the other hand, within the group of respondents that continued to work, home working seemed to be a protective factor. During this particular historical period, working from home was strictly associated with a reduced likelihood of contagion, and thus to a lower perception of risk. Moreover, the higher flexibility of the working schedule could encourage a better organization of the sleep/wake rhythms, favouring longer sleep duration. Consistently, the home workers slept almost half an hour more than those who continued to reach the workplace.

The changes in daily working time emerged as an essential predictor of sleep disturbances, and the increased work hours were associated with significant sleep problems. This result is consistent with other studies showing an adverse effect of the increased work routine on sleep quality and quantity[58, 59]. 
Interestingly, when working time was reduced, there was no benefit of home working. On the other hand, when the working schedule was maintained or increased, participants who worked remotely showed better sleep quality and fewer insomnia symptoms. However, when the home workers increased their daily working time, they reached the sleep disturbance level of the regular workers who

maintained/reduced the working hours. Therefore, working remotely during the current pandemic should be encouraged as a protective factor, focusing on avoiding extra working time. When the regular working day is not punctuated by fixed starting and ending time point, a common consequence could be the increase of daily working duration, with negative consequences on sleep.

To the best of our knowledge, the present is the most extensive investigation aimed at understanding the pandemic-related consequences on the general population's sleep. However, it should be acknowledged that we used a non-probabilistic sampling technique, with a higher representativity of the female gender and the young population.

The results confirmed the hypothesis that the lockdown due to the COVID-19 outbreak had significant repercussions on the sleep quality of the general population[3].

The restraining measures had a cumulative cost, and our results confirm the need to avoid over precautionary approaches, keeping the home confinement period as short as possible to limit its longterm negative consequences for sleep and mental health[60].

Our results are consistent with the current literature suggesting a higher predisposition of the female gender to develop sleep problems. In addition, the healthcare workers emerged again as an at-risk category. Moreover, our results showed that the differences in individual daily activity pattern preferences could represent a crucial predictor of sleep and psychological health during the pandemic period. We demonstrated a particular vulnerability of the ET people, while the circadian morning preference seems to be a protective factor during the current challenging period.

In light of this evidence, the vulnerable categories should be placed at the center of preventive interventions to avoid the exacerbation of sleep disturbances and mental health problems in the long run. Chronobiological interventions, such as melatonin, light exposure, and social rhythm regulation, could be effective strategies for ET people to hinder the onset or exacerbation of depression symptoms during the period of restraining measures[28].

In conclusion, we propose some guidelines for working during the COVID-19 pandemic. Individuals who suspended the working activity should maintain a regular daily activity to counteract the development and exacerbation of sleep disturbances. Home working should be encouraged, as long as the overall daily activity duration does not increase, establishing fixed starting and ending times of the workday. This aspect should be regulated since home working and teleworking could become increasingly widespread modalities regardless of the pandemic's conclusion[61]. In this view, the results of the present investigation could be generalizable to non-emergency periods. Furthermore, the subjects who work in telematic modality should avoid exposure to backlit screens of electronic devices before falling asleep 
since the increased evening exposure was suggested as a causal factor in developing sleep disturbances during lockdown[5].

An adequate sleep quality/quantity is essential to deal with stressful events[62] and preserve mental health[63], emotional regulation[64, 65], as well as the proper functioning of the immune system[66]. Consequently, the present results have a broad-spectrum of implications.

Our study's findings could be essential in the present period, where the second wave has become a reality, and hundreds of thousands of people are subjected to restraining measures worldwide. All the insights provided in this study should be considered from the institutions to design public campaigns aimed to promote sleep health and general well-being during the current unprecedented situation.

\section{Methods}

\section{Participants and experimental procedure}

A web-based survey has been disseminated through social media (Facebook, WhatsApp, Instagram, Twitter) from the third week to the end of the confinement period (25 March-3 May 2020), using a snowball sampling technique. A total of 13989 Italian citizens (mean age \pm standard deviation, $34.8 \pm$ 12.2 years, range $18-86,3223$ males) participated in the present investigation. The survey started with demographic questions (age, gender, education, occupation, geographic location) and COVID-related information (infection or forced quarantine). Demographic informations are reported in Table 4. Then, we asked to rate the perceived impact of the lockdown on sleep quality (positive, none, negative), and the occurred changes of bedtime (delayed, maintained, anticipated), wake-up time (delayed, unchanged, anticipated), and nap habits (increased, unchanged, reduced). Then, the survey comprised an evaluation of the working activity changes. In particular, we collected information on the suspension of the working activity (yes, no), the beginning of the home working modality (yes, no), and the changes of the daily working time (increased, unchanged, reduced). Subsequently, we evaluated sleep quality, insomnia severity symptoms, and chronotype, through a set of validated questionnaires: the Pittsburgh Sleep Quality Index (PSQI[6, 7]), the Insomnia Severity Index (ISI[8, 9]), the reduced version of the MorningnessEveningness Questionnaire (MEQr[10]). The PSQI is a 19-item questionnaire, and higher scores (range, 021) point to more severe sleep difficulties. Scores higher than 5 represent a valid indicator of poor sleep quality[6]. The ISI is a clinical instrument to assess the severity of insomnia symptoms. The score ranges from 0 to 28 and validated cut-off scores can be used to identify clinical insomnia conditions (0-7: no insomnia; 8-14 sub-threshold insomnia; 15-21: moderate insomnia; 22-28: severe insomnia) [8]. The MEQr is a 5-item questionnaire (range, 4-25) used to classify the circadian preference (ET: 4-10; NT: 11-18; MT: 19-25 score).

Finally, we assessed depression symptoms, perceived stress, and anxiety using (in order of presentation) the Beck Depression Inventory-second edition (BDI-II[11]), the 10-item Perceived Stress Scale (PSS-10[12]), and the state-anxiety subscale of the State-Trait Anxiety Inventory (STAI-X1[13]), respectively. The BDI-II is 
a 21-item depression symptoms inventory. Higher scores indicate more severe depression (range, 0-63). The PSS-10 is a 10-item questionnaire evaluating thoughts and feelings referred to stressful events. Higher scores point to higher perceived stress (range, $0-50$ ). The STAI-X1[13] is a 20-item scale included in the Cognitive Behavioural Assessment battery 2.0[67], used to evaluate anxiety. Higher scores point to more significant state anxiety. Participation in the entire survey required approximately 25 minutes, and the compilation of the last three questionnaires (BDI-II, 10-PSS, STAI-X1) was optional to avoid false/unreliable responses in the final part of the survey. A total of 9982 respondents $(71.4 \%)$ compiled the BDI-II, 9282 also the 10-PSS (66.5\%), and 9064 completed all the questionnaires (64.8\%).

The study was approved by the institutional review board of the University of L'Aquila (protocol $\mathrm{n}$. 43066/2020) and carried out according to the principles established by the Declaration of Helsinki. Online informed consent to participate in the research was obtained from all the respondents. 
Table 4

Demographic characteristics of the sample.

\begin{tabular}{|ll|}
\hline & N (\%) \\
\hline Age & \\
\hline $18-30$ & $7424(53.0)$ \\
\hline $31-50$ & $4755(33.9)$ \\
\hline Over 50 & $1810(12.9)$ \\
\hline Gender & \\
\hline Men & $3123(22.3)$ \\
\hline Women & $10866(77.6)$ \\
\hline Geographical location & \\
\hline Northern Italy & \\
\hline Central Italy & $5783(41.3)$ \\
\hline Southern Italy & $3389(24.2)$ \\
\hline Education & $4817(34.4)$ \\
\hline Middle school & $501(3.6)$ \\
\hline High school & $5350(38.2)$ \\
\hline Graduated & $6750(48.2)$ \\
\hline Over graduated & $1388(9.9)$ \\
\hline Occupation & \\
\hline Unemployed & $1347(9.6)$ \\
\hline Student & $4117(29.4)$ \\
\hline Worker & \\
\hline Healthcare work & $781(5.6)$ \\
\hline Other work & \\
\hline CoVID-19 infection & $(55.3)$ \\
\hline No & \\
\hline Yes & \\
\hline
\end{tabular}




\begin{tabular}{|ll|}
\hline & $\mathbf{N}(\%)$ \\
\hline No response & $144(1.0)$ \\
\hline Forced quarantine & \\
\hline No & $12890(92.1)$ \\
\hline Yes & $1032(7.4)$ \\
\hline No response & $67(0.5)$ \\
\hline
\end{tabular}

a Northern Italy: Aosta Valley, Emilia Romagna, Friuli-Venezia Giulia, Liguria, Lombardy, Piedmont, Trentino-Alto Adige, and Veneto. ${ }^{\mathrm{b}}$ Central Italy: Lazio, Marche, Tuscany, and Umbria. ${ }^{\mathrm{c}}$ Southern Italy: Abruzzo, Apulia, Basilicata, Calabria, Campania, Molise, Sardinia, and Sicily.

\section{Data analysis}

We excluded 814 participants from the PSQI score analyses due to compilation errors (i.e., respondents declared longer total sleep time compared with the reported total time in bed). We performed frequencies analyses to show the proportion of the reported impact of the lockdown period on sleep (negative, none, positive), and the changes of bedtime (anticipated, unchanged, delayed), wake-up time (anticipated, unchanged, delayed), and nap habits (increased, unchanged, reduced). According to the validated criteria of PSQI and ISI questionnaires, we calculated the prevalence of poor sleepers and clinical insomniacs within the entire sample.

Multiple regression analyses were carried out with PSQI and ISI scores as dependent variables. The regression models comprised the following continuous and categorical predictors: age, gender (man, woman), education (middle school, high school, graduated, over-graduated), occupation (healthcare work, other work, student, unemployed), geographic location (norther Italy, central Italy, southern Italy), the experience of the forced quarantine (yes, no, no response), duration of the confinement period (based on the day of participation to the survey), and the MEQr, BDI-II, PSS-10, and STAI-X1 questionnaire scores. We did not include the COVID-19 infection factor (yes, no, no response) due to the low number of infected subjects (only 44 participants).

We calculated the chronotype composition of our sample (MT, NT, ET) according to the MEQr cut-off. Then, we performed chi-square tests to evaluate the association of the three circadian typology groups with the perceived impact of the lockdown period on sleep, the reported changes of bedtime, wake-up time, and nap habits, and the prevalence of poor sleepers and clinical insomnia conditions.

To evaluate differences in sleep quality, insomnia severity symptoms, depression, perceived stress, and anxiety, between MT, NT, and ET, we carried out ANCOVAs on the scores of the five questionnaires (PSQI, ISI, BDI-II, PSS-10, STAI-X1), with chronotype (MT, NT, ET) as three-level between factor. The current 
literature supports a strong relationship between age and circadian preference[28,68]. Therefore, the analyses controlled for the effect of age used as a covariate.

Finally, we applied a t-test analysis to compare the PSQI and ISI scores of the respondents who suspended or maintained the working activity. In order to evaluate the effect on sleep quality and insomnia symptoms due to the changes in the modality (home working) and duration of the daily working activity, the PSQI and ISI scores were submitted to two-way ANOVAs, with "home working" (yes, no) and "daily working time" (increased, unchanged, reduced) as two-level and three-level between factors, respectively.

In some cases, further exploratory analyses were performed, using the information of interest (i.e., bedtime, wake-up time, sleep duration) derived from the PSQI (see "Results" paragraph).

All the analyses were two-tailed, and Bonferroni post hoc comparisons were performed in case of significant effects. P-value $<0.05$ was considered significant. When the data did not appear normally distributed or looked like heteroscedastic, "robust" or nonparametric techniques were used to check for bias in the inferential tests that could have led to misleading conclusions. Because these control analyses produced almost identical results to those obtained using the standard parametric tests, we concluded that violations of parametric assumptions were of negligible importance and we reported only the parametric test results.

\section{Declarations}

\section{Acknowledgements}

We want to thank all the Italians who participated in the present study. We are grateful to Jasmin Cascioli and Domenico Corigliano for their help in data collection.

\section{Author Contributions}

Conceptualization: F.S., M.F.; methodology: F.S., M.F.; investigation: F.S., G.A., L.V.; data curation: F.S.; Formal analysis: F.S., M.L.; writing - original draft: F.S.; Writing - review \& editing: F.S., M.L, A.D.A, D.T., M.F.; supervision: M.F.

\section{Competing Interests}

The authors declare no competing interests. This research did not receive any specific grant from funding agencies in the public, commercial, or not-for-profit sectors.

\section{Data Availability Statement}

The datasets analysed during the current study are available from the corresponding author on reasonable request. 


\section{References}

1. Rajkumar, R. P. COVID 19 and mental health: A review of the existing literature. Asian. J. Psychiatr. 52, 102066 https://doi.org/10.1016/j.ajp.2020.102066 (2020).

2. Vindegaard, N. \& Benros, M. E. COVID-19 pandemic and mental health consequences: systematic review of the current evidence. Brain Behav. Immun. 89, 531-542 (2020).

3. Altena, E. et al. Dealing with sleep problems during home confinement due to the COVID-19 outbreak: Practical recommendations from a task force of the European CBT-I Academy. J. Sleep Res. 29, e13052 https://doi.org/10.1111/jsr.13052 (2020).

4. Jahrami, H. et al. Sleep problems during COVID-19 pandemic by population: a systematic review and meta-analysis. J. Clin. Sleep Med. https://doi.org/10.5664/jcsm.8930 (2020).

5. Salfi, F. et al. Changes of evening exposure to electronic devices during the COVID-19 lockdown affect the time course of sleep disturbances. medRxiv. Preprint at. https://doi.org/10.1101/2020.10.20.20215756 (2020).

6. Buysse, D. J., Reynolds, C. F., Monk, T. H., Berman, S. R. \& Kupfer, D. J. The Pittsburgh sleep quality index: A new instrument for psychiatric practice and research. Psychiatry Res. 28, 193-213 (1989).

7. Curcio, G. et al. Validity of the Italian Version of the Pittsburgh Sleep Quality Index (PSQI). Neurol Sci. 34, 511-519 (2013).

8. Bastien, C. H., Vallière, A. \& Morin, C. M. Validation of the Insomnia Severity Index as an outcome measure for insomnia research. Sleep Med. 2, 297-307 (2001).

9. Castronovo, V. et al. Validation study of the Italian version of the Insomnia Severity Index (ISI). Neurol Sci. 37, 1517-1524 (2016).

10. Natale, V., Esposito, M. J., Martoni, M. \& Fabbri, M. Validity of the reduced version of the Morningness-Eveningness Questionnaire. Sleep Biol. Rhythms. 4, 72-74 (2006).

11. Ghisi, M., Flebus, G., Montano, A., Sanavio, E. \& Sica, C. Beck Depression Inventory-Second Edition. Adattamento italiano: Manuale (O-S Organizzazioni Speciali, Firenze, 2006).

12. Mondo, M., Sechi, C. \& Cabras, C. Psychometric evaluation of three versions of the Italian perceived stress scale. Curr. Psychol. https://doi.org/10.1007/s12144-019-0132-8 (2019).

13. Spielberger, C. D., Gorsuch, R. L. \& Lushene, R. E. The State-Trait Anxiety Inventory (STAI) Test Manual for Form X.(Consulting Psychologist Press, Palo Alto, 1970). Tr. it.: Lazzari, R. \& Pancheri, P. S.T.A.I. Questionario di autovalutazione dell'ansia di stato e di tratto. (Organizzazioni Speciali, Firenze, 1980).

14. Li, X. et al. Prevalence, risk factors, and clinical correlates of insomnia in volunteer and at home medical staff during the COVID-19. Brain Behav. Immun. 87, 140-141 (2020).

15. Wang, J. et al. Sleep disturbances among Chinese residents during the Coronavirus Disease 2019 outbreak and associated factors. Sleep Med. 74, 199-203 (2020).

16. Guadagni, V., Umiltà, A. \& laria, G. Sleep Quality, Empathy, and Mood During the Isolation Period of the COVID-19 Pandemic in the Canadian Population: Females and Women Suffered the Most. Front. 
Glob. Womens Health. 1, 585938 https://doi.org/10.3389/fgwh.2020.585938 (2020).

17. Casagrande, M., Favieri, F., Tambelli, R. \& Forte, G. The enemy who sealed the world: effects quarantine due to the COVID-19 on sleep quality, anxiety, and psychological distress in the Italian population. Sleep Med. 75, 12-20 (2020).

18. Cellini, N. et al. Changes in sleep timing and subjective sleep quality during the COVID-19 lockdown in Italy and Belgium: age, gender and working status as modulating factors. Sleep Med. 77, 112-119 (2021).

19. Muller, A. E. et al. The mental health impact of the covid-19 pandemic on healthcare workers, and interventions to help them: A rapid systematic review. Psychiatry Res. 293, 113441 https://doi.org/10.1016/j.psychres.2020.113441 (2020).

20. Pappa, S. et al. Prevalence of depression, anxiety, and insomnia among healthcare workers during the COVID-19 pandemic: a systematic review and meta-analysis. Behav. Immun. 88, 901-907 (2020).

21. Cellini, N., Canale, N., Mioni, G. \& Costa, S. Changes in sleep pattern, sense of time and digital media use during COVID-19 lockdown in Italy. J. Sleep Res. 29, e13074 https://doi.org/10.1111/jsr.13074 (2020).

22. Wang, W. et al. Sleep Disturbance and Psychological Profiles of Medical Staff and Non-Medical Staff During the Early Outbreak of COVID-19 in Hubei Province, China. Front. Psychiatry. 11, 733 https://doi.org/10.3389/fpsyt.2020.00733 (2020).

23. Leone, M. J., Sigman, M. \& Golombek, D. A. Effects of lockdown on human sleep and chronotype during the COVID-19 pandemic. Curr. Biol. 30, 930-931 https://doi.org/10.1016/j.cub.2020.07.015 (2020).

24. Korman, M. et al. COVID - 19-mandated social restrictions unveil the impact of social time pressure on sleep and body clock. Sci. Rep. 10, 22225 https://doi.org/10.1038/s41598-020-79299-7 (2020).

25. Wright, K. P. et al. Sleep in university students prior to and during COVID-19 Stay-at-Home orders. Curr. Biol. 30, 797-798 https://doi.org/10.1016/j.cub.2020.06.022 (2020).

26. Wittmann, M., Dinich, J., Merrow, M. \& Roenneberg, T. Social jetlag: misalignment of biological and social time. Chronobiol. Int. 23, 497-509 https://doi.org/10.1080/07420520500545979 (2006).

27. Kantermann, T. \& Behavior How a Global Social Lockdown Unlocks Time for Sleep. Curr. Biol. 30, 822-823 https://doi.org/10.1016/j.cub.2020.06.037 (2020).

28. Adan, A. et al. Circadian Typology: A Comprehensive Review. Chronobiol. Int. 29, 1153-1175 https://doi.org/10.3109/07420528.2012.719971 (2012).

29. Merikanto, I. et al. Relation of Chronotype to Sleep Complaints in the General Finnish Population. Chronobiol. Int. 29, 311-317 https://doi.org/10.3109/07420528.2012.655870 (2012).

30. Rique, G. L. N. et al. Relationship between chronotype and quality of sleep in medical students at the Federal University of Paraiba, Brazil. Sleep Sci. 7, 96-102 (2014). 
31. Fabbian, F. et al. Chronotype, gender and general health. Chronobiol. Int. 33, 863-882 https://doi.org/10.1080/07420528.2016.1176927 (2016).

32. Au, J. \& Reece, J. The relationship between chronotype and depressive symptoms: A meta-analysis. J. Affect. Disord. 218, 93-104 https://doi.org/10.1016/j.jad.2017.04.021 (2017).

33. Merikanto, I. et al. Evening types are prone to depression. Chronobiol. Int. 30, 719-725 https://doi.org/10.3109/07420528.2013.784770 (2013).

34. Fares, S. et al. Clinical correlates of chronotypes in young persons with mental disorders. Chronobiol. Int. 32, 1183-1191 https://doi.org/10.3109/07420528.2015.1078346 (2015).

35. Levandovski, R. et al. Depression scores associate with chronotype and social jetlag in a rural population. Chronobiol. Int. 28, 771-778 https://doi.org/10.3109/07420528.2011.602445 (2011).

36. Van den Berg, J. F., Kivelä, L. \& Antypa, N. Chronotype and depressive symptoms in students: An investigation of possible mechanisms. Chronobiol. Int. 35, 1248-1261 https://doi.org/10.1080/07420528.2018.1470531 (2018).

37. Kivelä, L., Papadopoulos, M. R. \& Antypa, N. Chronotype and Psychiatric Disorders. Curr. Sleep Medicine Rep. 4, 94-103 https://doi.org/10.1007/s40675-018-0113-8 (2018).

38. Simor, P., Zavecz, Z., Pálosi, V., Török, C. \& Köteles, F. The influence of sleep complaints on the association between chronotype and negative emotionality in young adults. Chronobiol. Int. 32, 1-10 https://doi.org/10.3109/07420528.2014.935786 (2015).

39. Rossi, R. et al. Mental Health Outcomes Among Frontline and Second-Line Health Care Workers During the Coronavirus Disease 2019 (COVID-19) Pandemic in Italy. JAMA Netw. Open. 3, e2010185 https://doi.org/10.1001/jamanetworkopen.2020.10185 (2020).

40. Madrid-Valero, J. J., Martínez-Selva, J. M., Ribeiro do Couto, B., Sánchez-Romera, J. F. \& Ordoñana, J. R. Age and gender effects on the prevalence of poor sleep quality in the adult population. Gaceta Sanitaria. 31, 18-22 (2017).

41. Mander, B. A., Winer, J. R. \& Walker, M. P. Sleep and Human Aging. Neuron. 94, 19-36 (2017).

42. Zhang, B. \& Wing, Y. K. Sex differences in insomnia: a meta-analysis. Sleep. 29, 85-93 (2006).

43. Kamel, N. S. \& Gammack, J. K. Insomnia in the Elderly: Cause, Approach, and Treatment. Am. J. Med. 119, 463-469 (2006).

44. Salfi, F. et al. Gender-related time course of sleep disturbances and psychological symptoms during the COVID-19 lockdown: A longitudinal study on the Italian population. Neurobiol. Stress. 13, 100259 https://doi.org/10.1016/j.ynstr.2020.100259 (2020).

45. Istituto Superiore di Sanità, 2020. Dati della Sorveglianza integrata COVID-19 in Italia. https://www.epicentro.iss.it/coronavirus/sars-cov-2-dashboard. (Accessed 1 November 2020).

46. SVIMEZ, 2020. SVIMEZ \& Rapporto, S. V. I. M. E. Z. 2020. https://Inx.svimez.info/svimez/presentazione-rapporto-svimez-2020/ (Accessed 28 November 2020). 
47. Wang, C. et al. A longitudinal study on the mental health of general population during the COVID-19 epidemic in China. Brain Behav. Immun. 87, 40-48 (2020).

48. Marelli, S. et al. Impact of COVID-19 lockdown on sleep quality in university students and administration staff. J. Neurol. https://doi.org/10.1007/s00415-020-10056-6 (2020).

49. Çetin, F. H. et al. Chronotypes and trauma reactions in children with ADHD in home confinement of COVID-19: full mediation effect of sleep problems. Chronobiol. Int. 37, 1214-1222 https://doi.org/10.1080/07420528.2020.1785487 (2020).

50. Baron, K. G. \& Reid, K. J. Circadian misalignment and health. Int. Rev. Psychiatry. 26, 139-154 https://doi.org/10.3109/09540261.2014.911149 (2014).

51. Jeon, H. J. \& Lee, S. J. Chronobiology and Sleep on Resilience. Chronobiol. Med. 1, 149-151 https://doi.org/10.33069/cim.2019.0026 (2019).

52. Antúnez, J. M., Navarro, J. F. \& Adan, A. Circadian typology is related to resilience and optimism in healthy adults. Chronobiol. Int. 32, 524-530 https://doi.org/10.3109/07420528.2015.1008700 (2015).

53. Lee, S. J. et al. Association between morningness and resilience in Korean college student. Chronobiol. Int. 33, 1391-1399 https://doi.org/10.1080/07420528.2016.1220387 (2016).

54. Romo-Nava, F. et al. The association between chronotype and perceived academic stress to depression in medical students. Chronobiol. Int. 33, 1359-1368 https://doi.org/10.1080/07420528.2016.1217230 (2016).

55. Hasler, B. P., Insana, S. P., James, J. A. \& Germain, A. Evening-type military veterans report worse lifetime posttraumatic stress symptoms and greater brainstem activity across wakefulness and REM sleep. Biol. Psychol. 94, 255-262 (2013).

56. Yun, J. A., Ahn, Y. S., Jeong, K. S., Joo, E. J. \& Choi, K. S. The relationship between chronotype and sleep quality in Korean firefighters. Clin. Psychopharmacol. Neurosci. 13, 201-208 (2015).

57. Zakay, D. Psychological time as information: the case of boredom. Front. Psychol. 5, 917 https://doi.org/10.3389/fpsyg.2014.00917 (2014).

58. Bidle, J. E. \& Hamermesh, D. S. Sleep and the Allocation of Time. J. Polit. Econ. 98, 922-943 (1990).

59. Virtanen, M. et al. Long working hours and sleep disturbances: the Whitehall II prospective cohort study. Sleep. 32, 737-745 (2009).

60. Brooks, S. K. et al. The psychological impact of quarantine and how to reduce it: rapid review of the evidence. Lancet. 395, 912-920 (2020).

61. European commission, 2020. Telework in the EU before and after the COVID-19: where we were, where we head to. https://ec.europa.eu/jrc/sites/jrcsh/files/jrc120945_policy_brief__covid_and_telework_final.pdf. (Accessed 28 November 2020).

62. Leggett, A., Burgard, S. \& Zivin, K. The Impact of Sleep Disturbance on the Association Between Stressful Life Events and Depressive Symptoms. J. Gerontol. B. Psychol. Sci. Soc. Sci. 71, 118-128 (2016). 
63. Pigeon, W. R., Bishop, T. M. \& Krueger, K. M. Insomnia as a Precipitating Factor in New Onset Mental Illness: a Systematic Review of Recent Findings. Curr. Psychiatry Rep. 19, 44 https://doi.org/10.1007/s11920-017-0802-x (2017).

64. Tempesta, D., Socci, V., De Gennaro, L. \& Ferrara, M. Sleep and emotional processing. Sleep Med. Rev. 40, 183-195 (2018).

65. Tempesta, D., Salfi, F., De Gennaro, L. \& Ferrara, M. The impact of five nights of sleep restriction on emotional reactivity. J. Sleep Res. 29, e13022 https://doi.org/10.1111/jsr.13022 (2020).

66. Bryant, P. A., Trinder, J. \& Curtis, N. Sick and tired: Does sleep have a vital role in the immune system?. Nat. Rev. Immunol. 4, 457-467 (2004).

67. Sanavio, E., Bertolotti, G., Michelin, P., Vidotto, G. \& Zotti, A. M. CBA 2.0 - Cognitive Behavioural Assessment 2.0 - Scale Primarie: Manuale (Organizzazioni Speciali, Firenze, 1997).

68. Carrier, J., Monk, T. H., Buysse, D. J. \& Kupfer, D. J. Sleep and morningness-eveningness in the 'middle' years of life (20-59 y). J. Sleep Res. 6, 230-237 (2005).

\section{Figures}

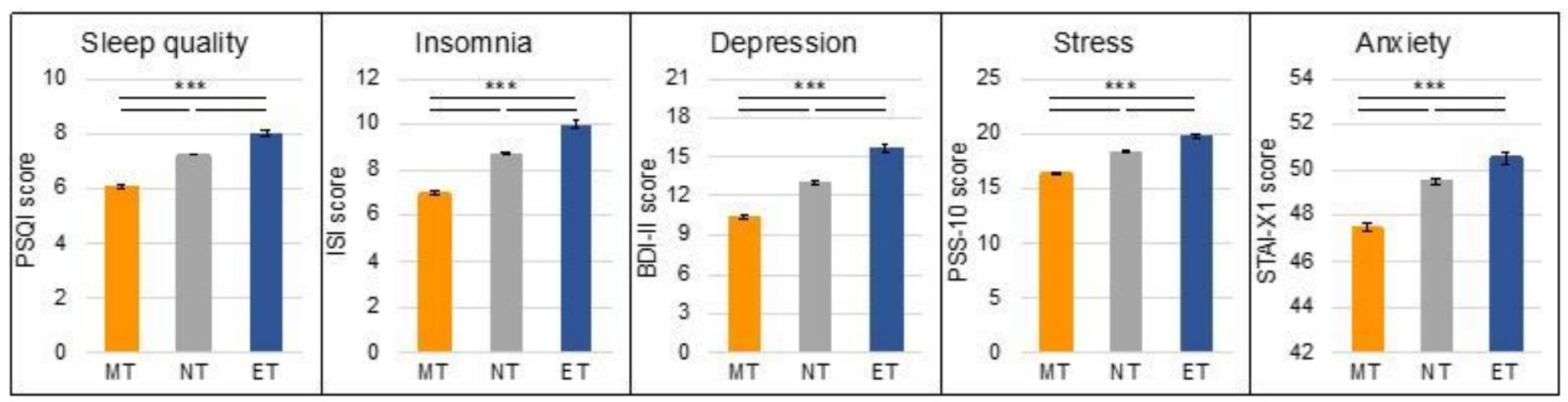

\section{Figure 1}

Sleep quality (PSQI), insomnia severity symptoms (ISI), depression (BDI-II), perceived stress (PSS-10), and anxiety (STAI-X1) for the three chronotype groups (Morning Type - MT, Neither Type - NT, Evening Type ET). Means (and standard errors) of questionnaire scores are reported. Results of Bonferroni post hoc comparisons are reported with asterisks $\left({ }^{\star * *} \mathrm{P}<0.001\right)$. 


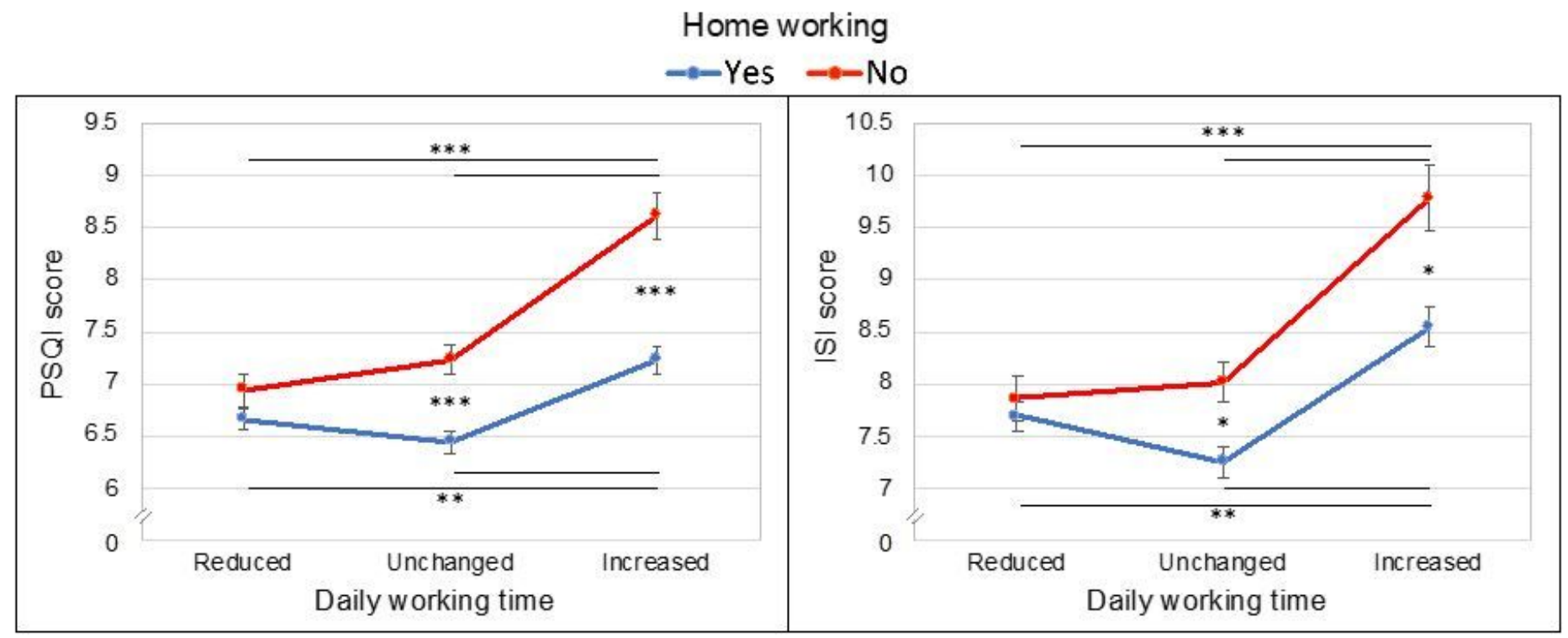

Figure 2

Interaction between "home working" (yes, no) and "daily working time" (reduced, unchanged, increased) factors, for sleep quality (PSQI) and insomnia severity symptoms scores (ISI). The figures report means (and standard errors) of the scores of the PSQI and ISI questionnaires. Results of Bonferroni post hoc comparisons are reported with asterisks $\left({ }^{\star} \mathrm{P}<0.05 ;{ }^{*} \mathrm{P}<0.01{ }^{\star \star \star} \mathrm{P}<0.001\right)$. 